\title{
Monte Carlo Simulation for Computing the Worst Value of the Objective Function in the Interval Linear Programming
}

\author{
M. Allahdadi ${ }^{1} \cdot$ A. Khaje Golestane ${ }^{1}$
}

Published online: 1 July 2015

C) Springer India Pvt. Ltd. 2015

\begin{abstract}
In this paper, we consider the interval linear programming (ILP) with equality constraints. Computing the best value of the objective function is easy, but obtaining the worst value of the objective function is much more complicated. Firstly, we determine range of optimal values of the objective function. Secondly, by considering some distribution functions, we use Monte Carlo simulation to explore the solutions for the ILP model, and then we compare the results obtained through the simulations.
\end{abstract}

Keywords Interval linear programming - Monte Carlo simulation · The worst optimal value

\section{Introduction}

One of the basic tools for description uncertain problems existing in real world is interval linear programming (ILP). Many methods have been proposed for solving ILP models [1-32]. Some methods give range of optimal values of the ILP models $[4,10,11,26]$. Some of them are focused on interval arithmetic and extensions of the simplex algorithm [3,17,18,24]. Some of methods are based on basis stability $[9,19,25,29]$. Under the assumption of basis stability, we can determine range of optimal values. In some of other methods, two sub-models with deterministic parameters were formulated. By solving the two sub-models, solutions for the optimized interval objective and interval decision variables can be obtained [13,16,31,32].

The best and the worst method (BWC) proposed by Tong converts ILP model into two sub-models [31]. Chinneck and Ramadan extended the BWC method to include nonnegative variables and equality constraints [4]. Huang and Moore proposed a new interval linear programming method (ILP) [16]. This method incorporates interval information directly into the optimization process.

M. Allahdadi

m_allahdadi@math.usb.ac.ir

1 Mathematics Faculty, University of Sistan and Baluchestan, Zahedan, Iran 
An interval number $x^{ \pm}$is generally represented as $\left[x^{-}, x^{+}\right]$where $x^{-} \leq x^{+}$. If $x^{-}=x^{+}$, then $x^{ \pm}$will be degenerate.

If $A^{-}$and $A^{+}$are two matrices in $\mathbb{R}^{m \times n}$ and $A^{-} \leq A^{+}$, then the set of matrices

$$
\mathbf{A}^{ \pm}=\left[A^{-}, A^{+}\right]=\left\{A \mid A^{-} \leq A \leq A^{+}\right\}
$$

is called an interval matrix, and the matrices $A^{-}$and $A^{+}$are called its bounds. Center and radius matrices have been defined as

$$
A^{c}=\frac{1}{2}\left(A^{+}+A^{-}\right), \quad A^{\Delta}=\frac{1}{2}\left(A^{+}-A^{-}\right) .
$$

A square interval matrix $\mathbf{A}^{ \pm}$is called regular if each $A \in \mathbf{A}^{ \pm}$is nonsingular.

A special case of an interval matrix is an interval vector which is a one-column interval matrix

$$
\mathbf{x}^{ \pm}=\left\{\mathbf{x} \mid \mathbf{x}^{-} \leq \mathbf{x} \leq \mathbf{x}^{+}\right\}
$$

where $\mathbf{x}^{-}, \mathbf{x}^{+} \in \mathbb{R}^{n}$.

Interval arithmetic is defined in [1].

We consider ILP model as follows:

$$
\begin{array}{ll}
\min & z^{ \pm}=\sum_{j=1}^{n} c_{j}^{ \pm} x_{j}^{ \pm} \\
\text {s.t. } & \sum_{j=1}^{n} a_{i j}^{ \pm} x_{j}^{ \pm}=b_{i}^{ \pm}, \quad i=1,2, \ldots, m \\
& x_{j}^{ \pm} \geq 0, \quad j=1,2, \ldots, n .
\end{array}
$$

By

$$
\begin{array}{ll}
\min & z=\sum_{j=1}^{n} c_{j} x_{j} \\
\text { s.t. } & \sum_{j=1}^{n} a_{i j} x_{j}=b_{i}, \quad i=1,2, \ldots, m \\
& x_{j} \geq 0, \quad j=1,2, \ldots, n .
\end{array}
$$

we denote characteristic model of the ILP model (1), where $a_{i j} \in a_{i j}^{ \pm}, c_{j} \in c_{j}^{ \pm}$and $b_{i} \in b_{i}^{ \pm}$. Optimal solution set of the ILP is defined as a set of all optimal solutions over all characteristic models.

The interval $\left[z_{\text {opt }}^{-}, z_{\text {opt }}^{+}\right]$is called the range of the optimal values of the ILP model (1). Computing of the best value $z_{\text {opt }}^{-}$is easy, but obtaining the worst value $z_{\text {opt }}^{+}$is NP-hard [5].

In this paper, we use Monte Carlo simulation when all of the interval coefficients of the ILP are replaced by the confidence interval of a random variable with normal, uniform and beta distributions. Obtaining the worst value of the objective function of the ILP model is considered. Also, we determine the optimal solutions under the distributions and then we compare the solutions obtained through the Monte Carlo simulation with the exact solutions.

\section{Range of the Optimal Values}

In this section, we review some notations and the method for obtaining the best and the worst values of the objective function of the ILP model (1) [5].

Let $Y_{m}$ be the set of all $\{-1,1\}$ m-dimensional vectors, i.e.

$$
Y_{m}=\left\{\mathbf{y} \in \mathbb{R}^{m}|| \mathbf{y} \mid=e\right\},
$$


where $e=(1,1, \ldots, 1)^{T}$. For a given $\mathbf{y} \in\{ \pm 1\}^{m}$, by

$$
T_{\mathbf{y}}=\operatorname{diag}\left(y_{1}, y_{2}, \ldots, y_{m}\right),
$$

we denote the corresponding diagonal matrix. For each $\mathbf{x} \in \mathbb{R}^{n}$, we denote its sign vector $\operatorname{sign}(\mathbf{x})$ by

$$
(\operatorname{sign} \mathbf{x})_{i}=\left\{\begin{array}{cc}
1 & x_{i} \geq 0 \\
-1 & x_{i}<0,
\end{array}\right.
$$

where $1,2, \ldots, n$. Then we have $|\mathbf{x}|=T_{z} \mathbf{x}$, where $\mathbf{z}=\operatorname{sign}(\mathbf{x}) \in Y_{n}$.

Consider an interval matrix $\mathbf{A}^{ \pm}=\left[A^{-}, A^{+}\right]=\left[A^{c}-A^{\Delta}, A^{c}+A^{\Delta}\right]$ and an interval vector $\mathbf{b}^{ \pm}=\left[b^{-}, b^{+}\right]=\left[b^{c}-b^{\Delta}, b^{c}+b^{\Delta}\right]$. For each $\mathbf{y} \in Y_{m}$ and $\mathbf{z} \in Y_{n}, A_{\mathbf{y z}}$ and $b_{\mathbf{y}}$ are defined as follows [5].

$$
\begin{aligned}
A_{\mathbf{y z}} & =A^{c}-T_{\mathbf{y}} A^{\Delta} T_{\mathbf{z}}, \\
b_{\mathbf{y}} & =b^{c}+T_{\mathbf{y}} b^{\Delta} .
\end{aligned}
$$

Theorem 1 [5] The best and the worst values of the objective function of the ILP model (1) could be obtained as follows.

$$
\begin{aligned}
& z_{o p t}^{-}=\inf \left\{c^{-T} \mathbf{x} \mid A^{-} \mathbf{x} \leq b^{+}, A^{+} \mathbf{x} \geq b^{-}, \mathbf{x} \geq 0\right\}, \\
& z_{o p t}^{+}=\sup _{\mathbf{y} \in Y_{m}} f\left(A_{\mathbf{y e}}, b_{\mathbf{y}}, c^{+}\right),
\end{aligned}
$$

where $f(A, b, c)=\inf \left\{c^{T} \mathbf{x} \mid A \mathbf{x}=b, \mathbf{x} \geq 0\right\}$.

Theorem 2 [5] Computing the worst value of the objective function of the ILP model (1) is NP-hard.

While computing $z_{\text {opt }}^{-}$is easy, computation of $z_{o p t}^{+}$is much more involved [5].

Example 1 Consider the interval linear programming

$$
\begin{array}{ll}
\min & z=x_{1}^{ \pm}+x_{2}^{ \pm} \\
\text {s.t. } & {[2,3] x_{1}^{ \pm}+x_{2}^{ \pm}=[3,4]} \\
& {[4,6] x_{1}^{ \pm}+[-3,-2] x_{2}^{ \pm}=[1,5]} \\
& x_{1}^{ \pm}, x_{2}^{ \pm} \geq 0 .
\end{array}
$$

By Theorem 1, the best value of the objective function is determined by solving the following model.

$$
\begin{array}{cl}
\min & z^{-}=x_{1}+x_{2} \\
\text { s.t. } & 2 x_{1}+x_{2} \leq 4 \\
& 4 x_{1}-3 x_{2} \leq 5 \\
& 3 x_{1}+x_{2} \geq 3 \\
& 6 x_{1}-2 x_{2} \geq 1 \\
& x_{1}, x_{2} \geq 0 .
\end{array}
$$

The optimal value is $z_{o p t}^{-}=1$. The worst value of the objective function is $z_{o p t}^{+}=3.1$ which results from solving the following model. 


$$
\begin{array}{ll}
\min & z^{+}=x_{1}+x_{2} \\
\text { s.t. } & 2 x_{1}+x_{2}=4 \\
& 6 x_{1}-2 x_{2}=1 \\
& x_{1}, x_{2} \geq 0 .
\end{array}
$$

Therefore, range of optimal values of the objective function is [1, 3.1].

\section{Monte Carlo Simulation}

A Monte Carlo method is used to evaluation solving methods [30]. We use the simulation for example 1 based on three distribution functions, normal, uniform and beta. We compare the solution obtained through the distribution functions with the exact solution. In view of the solutions resulted from the simulations, we determine coverage rate (CR) and validity rate (VR) proposed by Lu et al. [20].

Firstly, we generate 15000 Monte Carlo samples for each coefficient of the ILP model (5). We simulate three probability density functions for each coefficient of the ILP model (5). For a random variable with normal distribution $N(\mu, \sigma)$, the effective range can be considered as $[\mu-3 \sigma, \mu+3 \sigma][30]$.

For example, for $a_{11}=$ [2,3], Figure 1 shows three samples of normal, uniform and beta distributions. Values of $z$ resulted from samples of the distributions are shown in Figs. 2, 3 and 4.

Coverage rate $(\mathrm{CR})$ and valid rate (VR) proposed by $\mathrm{Lu}$ et al. are used as indicators to qualify the performance. The coverage and valid rates of the interval solution $\mathbf{x}$ are defined as follows [20]:

$$
\begin{aligned}
& C R\left(\mathbf{x}^{ \pm}\right)=\frac{\text { The number of samples whose values lie in } \mathbf{x}^{ \pm}}{\text {Total sample used in simulation }} \times 100 \% \\
& V R\left(\mathbf{x}^{ \pm}\right)=\frac{\text { The width of intersection between effective range and } \mathbf{x}^{ \pm}}{\text {The width of } \mathbf{x}^{ \pm}} \times 100 \%
\end{aligned}
$$
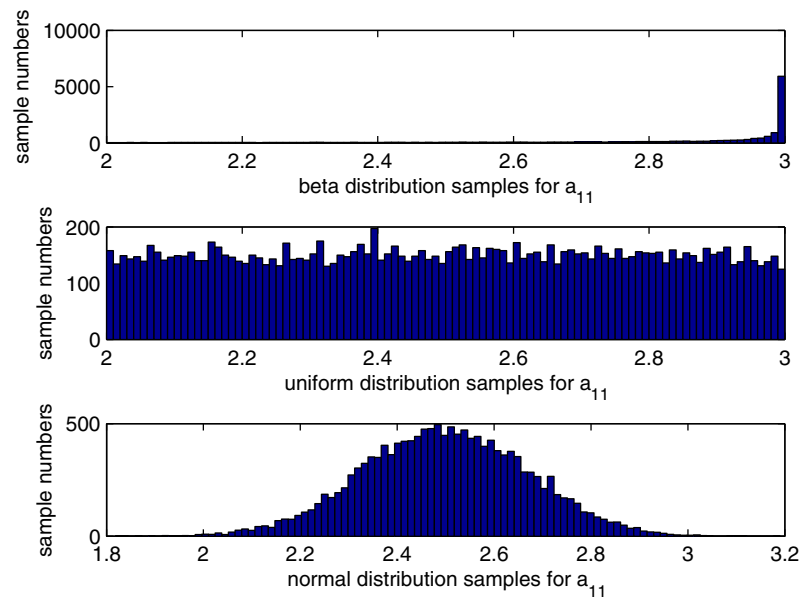

Fig. 1 Monte Carlo simulation for $a_{11}$ of the ILP model (5) 


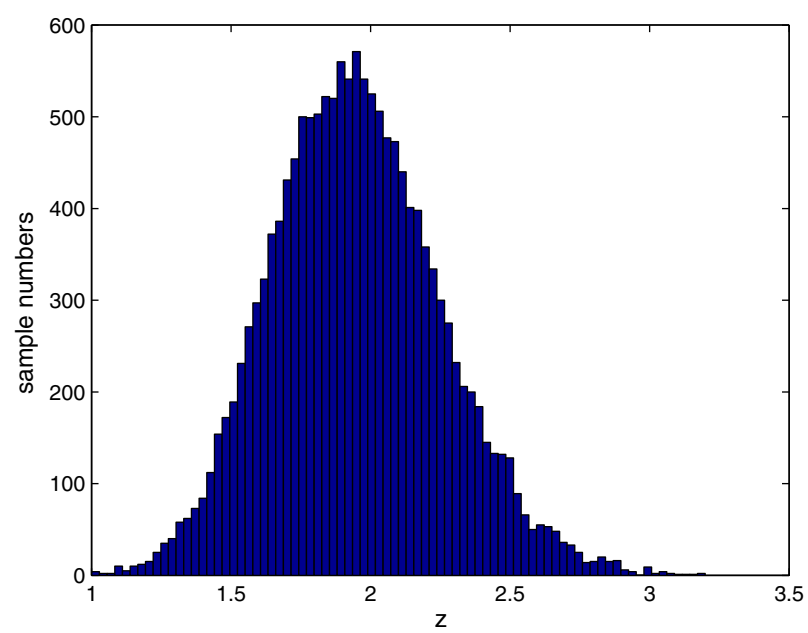

Fig. 2 Values of $z$ resulted from Monte Carlo simulation for normal distribution of the ILP model (5)

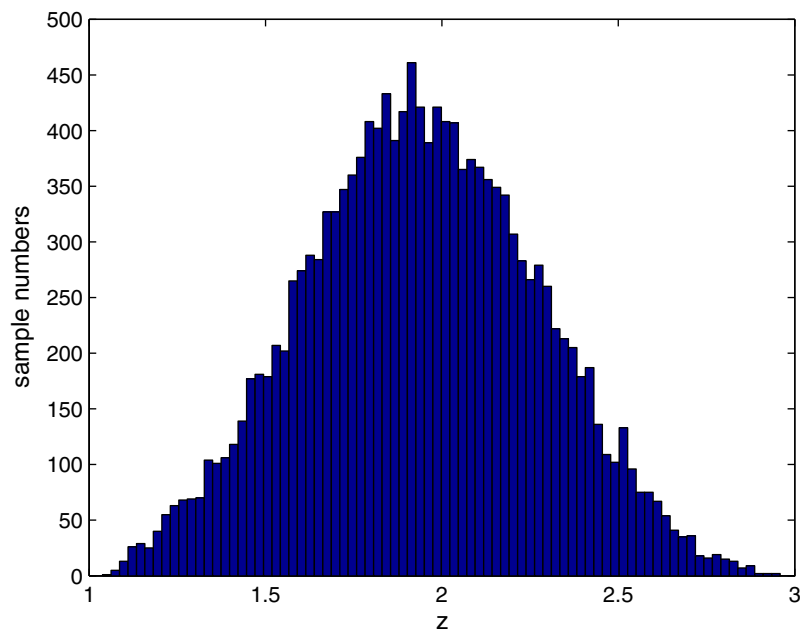

Fig. 3 Values of $z$ resulted from Monte Carlo simulation for uniform distribution of the ILP model (5)

The larger the CR value, the higher the chance of the exact solution lie in the interval of solutions. The higher the VR value of the solutions, the more valid information is included in the solutions [20].

Suppose all coefficients of the ILP model (5) have normal distribution. The valid rate of $z$ can be obtained as follows:

$$
\begin{aligned}
V R\left(z^{ \pm}=[1,3.1]\right) & =\frac{\text { The width of }\{[1,3.1] \cap[1.0394,2.8585]\}}{\text { The width of }[1,3.1]} \times 100 \% \\
& =86.62 \%,
\end{aligned}
$$

which the interval $[1.0394,2.8585]$ is interval obtained through simulation for normal distribution. 


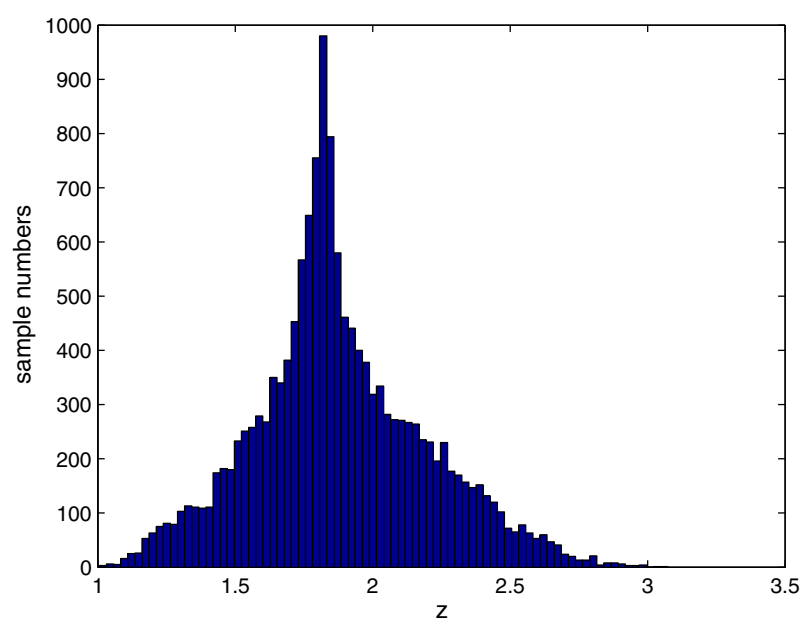

Fig. 4 Values of $z$ resulted from Monte Carlo simulation for beta distribution of the ILP model (5)

Table 1 Results for the three distribution samples of the ILP model (5)

\begin{tabular}{lll}
\hline Distribution & $z$ & $V R(z)(\%)$ \\
\hline Normal & {$[1.0394,2.8585]$} & 86.62 \\
Uniform & {$[0.9512,2.9298]$} & 93.89 \\
Beta & {$[0.9384,2.8186]$} & 89.53 \\
\hline
\end{tabular}

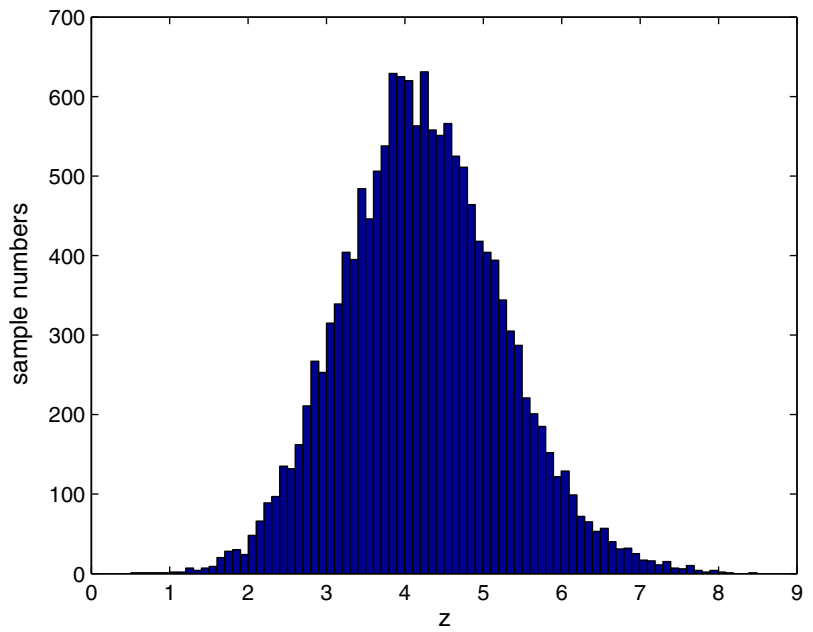

Fig. 5 Values of $z$ resulted from Monte Carlo simulation for normal distribution of the ILP model (6)

The values of $\operatorname{VR}(z)$ for the uniform and beta distributions are 93.89 and $89.53 \%$, respectively. Therefore, the samples of uniform distribution are better than the others.

The simulation results for the three samples of distributions are presented in Table 1. 


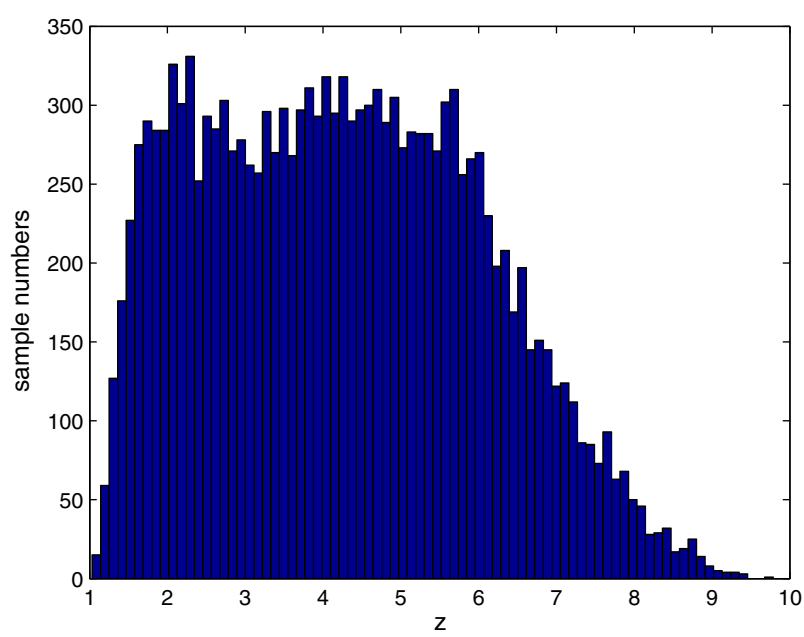

Fig. 6 Values of $z$ resulted from Monte Carlo simulation for uniform distribution of the ILP model (6)

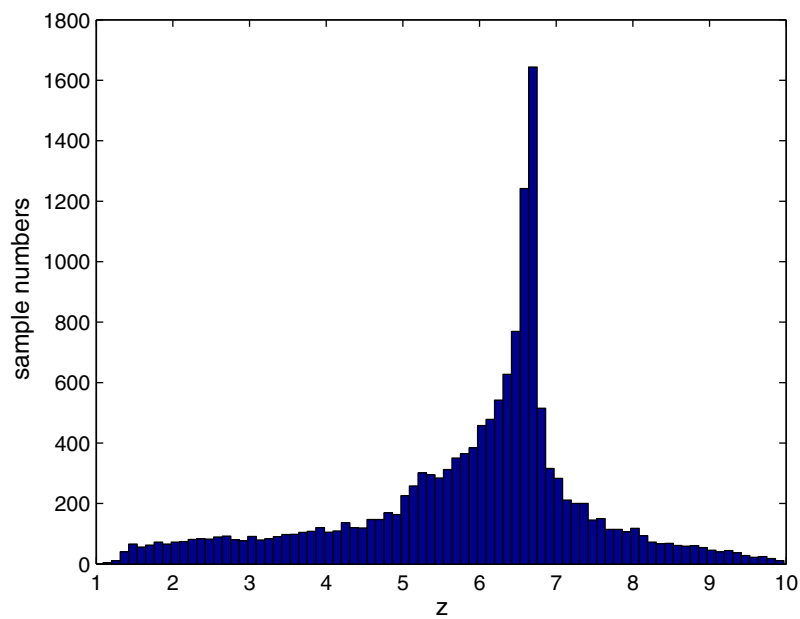

Fig. 7 Values of $z$ resulted from Monte Carlo simulation for beta distribution of the ILP model (6)

Example 2 Consider the interval linear programming

$$
\begin{array}{ll}
\min & z=[1,5] x_{1}^{ \pm}+[3,4] x_{2}^{ \pm} \\
\text {s.t. } & {[2,3] x_{1}^{ \pm}+x_{2}^{ \pm}=[3,4]} \\
& x_{1}^{ \pm}, x_{2}^{ \pm} \geq 0 .
\end{array}
$$

By Theorem 1, range of the optimal values of the objective function is [1, 10]. Values of $z$ resulted from samples of the distributions are shown in Figs. 5, 6 and 7. The simulation results for the samples of distributions are presented in Table 2. By attention to the values of $V R(z)$ for three samples of distribution, the samples of beta distribution and then uniform distribution are better than the samples of normal. 
Table 2 Results for the three distribution samples of the ILP model (6)

\begin{tabular}{lll}
\hline Distribution & $z$ & $V R(z)(\%)$ \\
\hline Normal & {$[1.2031,7.2702]$} & 67.41 \\
Uniform & {$[1.0526,9.5641]$} & 94.57 \\
Beta & {$[1.1724,10.6192]$} & 98.08 \\
\hline
\end{tabular}

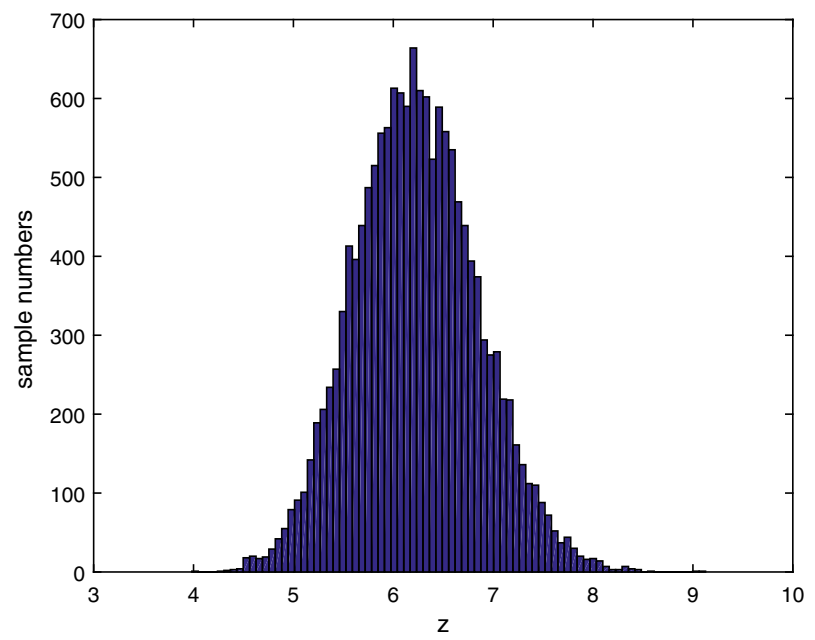

Fig. 8 Values of $z$ resulted from Monte Carlo simulation for normal distribution of the ILP model (7)

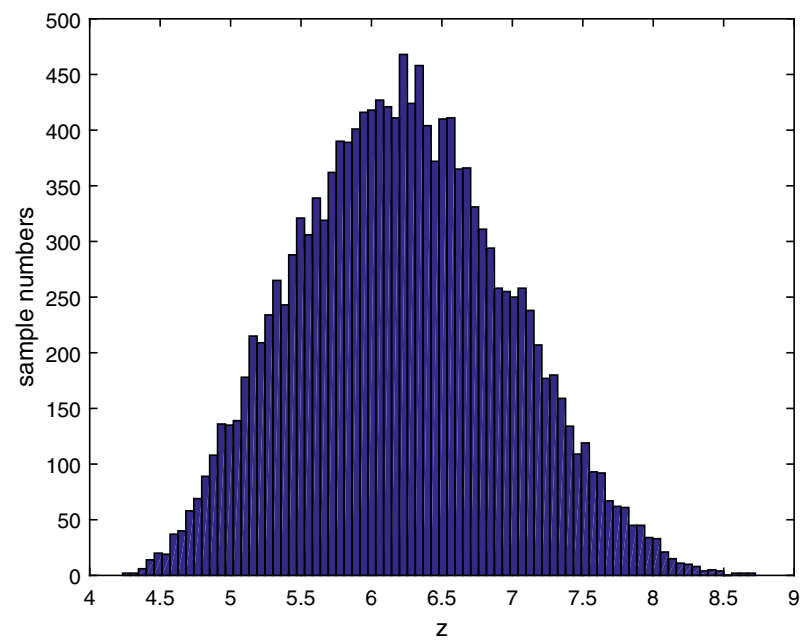

Fig. 9 Values of $z$ resulted from Monte Carlo simulation for uniform distribution of the ILP model (7)

Example 3 Consider the interval linear programming

$$
\begin{array}{ll}
\min & z=[2,4] x_{1}^{ \pm}+[5,7] x_{2}^{ \pm}+[2,3] x_{3}^{ \pm} \\
\text {s.t. } & -2 x_{1}^{ \pm}+[4,5] x_{2}^{ \pm}+[5,6] x_{3}^{ \pm}=6 \\
& {[5,6] x_{1}^{ \pm}-4 x_{2}^{ \pm}+[1,2] x_{3}^{ \pm}=7} \\
& x_{1}^{ \pm}, x_{2}^{ \pm}, x_{3}^{ \pm} \geq 0 .
\end{array}
$$




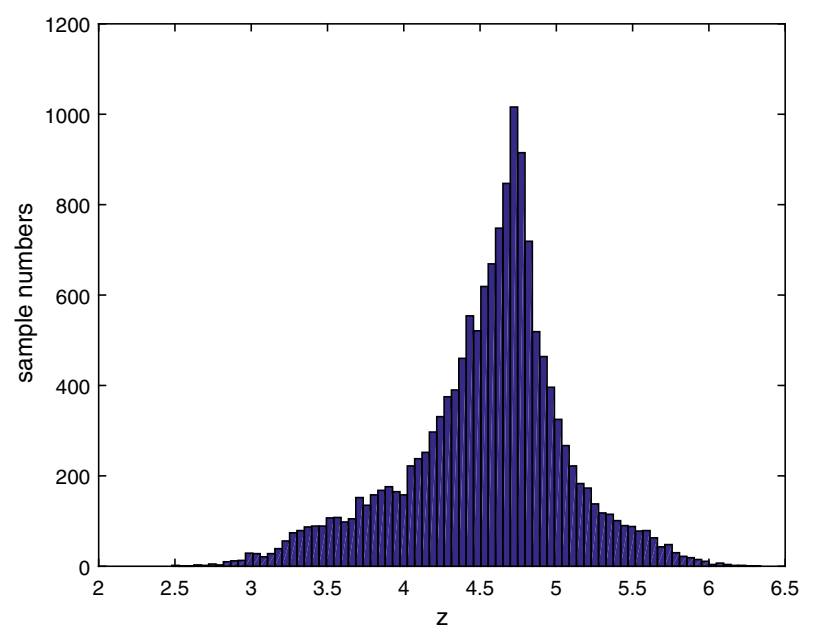

Fig. 10 Values of $z$ resulted from Monte Carlo simulation for beta distribution of the ILP model (7)

Table 3 Results for the three distribution samples of the ILP model (7)

\begin{tabular}{lll}
\hline Distribution & $z$ & $V R(z)(\%)$ \\
\hline Normal & {$[4.3818,8.0844]$} & 71.41 \\
Uniform & {$[3.9962,8.4461]$} & 85.82 \\
Beta & {$[2.9939,6.1016]$} & 59.93 \\
\hline
\end{tabular}

By Theorem 1, range of the optimal values of the objective function is [4, 9.1852]. Values of $z$ obtained through samples of the distributions are shown in Figs. 8, 9 and 10. The simulation results for the samples of distributions are presented in Table 3. By attention to the values of $V R(z)$ for three samples of distribution, the samples of uniform distribution are better than the others.

\section{Conclusion}

Computing the worst value of the objective function is much more complicated for ILP models with equality constraints. By considering some distribution functions, Monte Carlo simulation has been used to explore the values of the objective function. The results show that when the range of the objective function needs to be obtained, uniform and beta distribution samples for all coefficients of the ILP model are applicable.

Acknowledgments We would like to thank anonymous referees for their constructive comments and suggestions that helped to improve the paper.

\section{References}

1. Alefeld, G., Herzberger, J.: Introduction to Interval Computations. Academic Press, New York (1983)

2. Allahdadi, M., Mishmast Nehi, H.: The optimal solutions set of the interval linear programming problems. Optim. Lett. 7, 893-1911 (2013)

3. Beeck, H.: Linear programming with inexact data. Technical report TUM-ISU-7830, Technical University of Munich, Munich (1978) 
4. Chinneck, J.W., Ramadan, K.: Linear programming with interval coefficients. J. Oper. Res. Soc. 51, 209-220 (2000)

5. Fiedler, M., Nedoma, J., Ramik, J., Zimmermann, K.: Linear Optimization Problems With Inexact Data. Springer, New York (2003)

6. Guo, P., Huang, G.H., He, L., Li, H.L.: Interval-parameter fuzzy-stochastic semi-infinite mixed-integer linear programming for waste management under uncertainty. Environ. Model. Assess. 14, 521-537 (2009)

7. He, L., Huang, G., Zeng, G., Lu, H.: Fuzzy inexact mixed-integer semiinfinite programming for municipal solid waste management planning. J. Environ. Eng. 134, 572-581 (2008)

8. He, L., Huang, G.H., Zeng, G.M., Lu, H.W.: Identifying optimal regional solid waste management strategies through an inexact integer programming model containing infinite objectives and constraints. Waste Manag. 29, 21-31 (2009)

9. Hladik, M.: How to determine basis stability in interval linear programming. Optim. Lett. 8, 375-389 (2014)

10. Hladik, M.: Interval linear programming: a survay. In: Mann, Z.A. (ed.) Linear Programming New Frontiers, pp. 1-46. Nova Science publishers Inc., New York (2012)

11. Hladik, M.: On approximation of the best case optimal value in interval linear programming. Optim. Lett. 8, 1985-1997 (2014)

12. Huang, G.H., Baetz, B.W., Patry, G.G.: A grey linear programming approach for municipal solid waste management planning under uncertainty. Civ. Eng. Environ. Syst. 9, 319-335 (1992)

13. Huang, G.H., Baetz, B.W., Patry, G.G.: Grey integer programming: an application to waste management planning under uncertainty. Eur. J. Oper. Res. 83, 594-620 (1995)

14. Huang, G.H., Baetz, B.W., Patry, G.G.: Trash-flow allocation: planning under uncertainty. Interfaces $\mathbf{2 8}$, 36-55 (1998)

15. Huang, G.H., Cao, M.F.: Analysis of solution methods for interval linear programming. J. Environ. Inform. 17(2), 54-64 (2011)

16. Huang, G.H., Moore, R.D.: Grey linear programming, its solving approach, and its application. Inter. J. Syst. Sci. 24, 159-172 (1993)

17. Jansson, C.: A self-validating method for solving linear programming problems with interval input data. In: Kulisch, U., Stetter, H.J. (eds.) Scientific computation with automatic result verification. Computing Suppl., 6 pp. 33-45 (1988)

18. Jansson, C., Rump, S.M.: Rigorous solution of linear programming problems with uncertain data. Z. Oper. Res. 35(2), 87-111 (1991)

19. Konickova, J.: Sufficient condition of basis stability of an interval linear programming problem. Z. Angew. Math. Mech. 81(3), 677-678 (2001)

20. Lu, H.W., Cao, M.F., Wang, Y., Fan, X., He, L.: Numerical solutions comparison for interval linear programming problems based on coverage and validity rates. Appl. Math. Model. 38, 1092-1100 (2014)

21. Lu, H.W., Huang, G.H., He, L.: A semi-infinite analysis-based inexact two-stage stochastic fuzzy linear programming approach for water resources management. Eng. Opt. 41, 73-85 (2009)

22. Lu, H.W., Huang, G.H., Liu, L., He, L.: An interval-parameter fuzzy-stochastic programming approach for air quality management under uncertainty. Environ. Eng. Sci. 25, 895-909 (2008)

23. Lu, H.W., Huang, G.H., Liu, Z.F., He, L.: Greenhouse gas mitigation-induced rough-interval programming for municipal solid waste management. J. Air Waste Manag. Assoc. 58, 1546-1559 (2008)

24. Machost, B.: Numerische Behandlung des Simplexverfahrens mit intervallanalytischen Methoden. Tech. Rep. 30, Berichte der Gesellschaft fr Mathematik und Datenverarbeitung, 54 p., Bonn (1970)

25. Mraz, F.: On infimum of optimal objective function values in interval linear programming. Technical report KAM Series, pp. 96-337, Department of Applied Mathematics, Charles University, Prague (1996)

26. Mraz, F.: Calculating the exact bounds of optimal values in LP with interval coefficients. Ann. Oper. Res. 81, 51-62 (1998)

27. Rex, J., Rohn, J.: Sufficient conditions for regularity and singularity of interval matrices. SIAM J. Matrix Anal. Appl. 20(2), 437-445 (1998)

28. Rohn, J.: Cheap and tight bound: The recent result by E. Hansen can be made more efficient. Interval Comput. 4, 13-21 (1993)

29. Rohn, J.: Stability of the optimal basis of a linear program under uncertainty. Oper. Res. Lett. 13(1), 912 (1993)

30. Tierney, L., Mira, A.: Some adaptive Monte Carlo methods for Bayesian inference. Stat. Probab. Lett. 18, 2507-2515 (1999)

31. Tong, S.C.: Interval number, fuzzy number linear programming. Fuzzy Sets Syst. 66, 301-306 (1994)

32. Zhou, F., Huang, G.H., Chen, G., Guo, H.: Enhanced-interval linear programming. Eur. J. Oper. Res. 199, 323-333 (2009) 